\title{
Identifying Achilles-heel roads in real-sized networks
}

\author{
Saeed Asadi Bagloee ${ }^{1}$ (D) Majid Sarvi $^{1} \cdot$ Russell George Thompson $^{2} \cdot$ \\ Abbas Rajabifard ${ }^{3}$
}

Received: 26 August 2016/Revised: 14 December 2016/Accepted: 29 December 2016/Published online: 13 February 2017

(C) The Author(s) 2017. This article is published with open access at Springerlink.com

\begin{abstract}
Ensuring a minimum operational level of road networks in the presence of unexpected incidents is becoming a hot subject in academic circles as well as industry. To this end, it is important to understand the degree to which each single element of the network contributes to the operation and performance of a network. In other words, a road can become an "Achilles-heel" for the entire network if it is closed due to a simple incident. Such insight of the detrimental loss of the closure of the roads would help us to be more vigilant and prepared. In this study, we develop an index dubbed as Achilles-heel index to quantify detrimental loss of the closure of the respective roads. More precisely, the Achilles-heel index indicates how many drivers are affected by the closure of the respective roads (the number of affected drivers is also called travel demand coverage). To this end,
\end{abstract}

Saeed Asadi Bagloee

saeed.bagloee@unimelb.edu.au

Majid Sarvi

majid.sarvi@unimelb.edu.au

Russell George Thompson

rgthom@unimelb.edu.au

Abbas Rajabifard

abbas.r@unimelb.edu.au

1 Smart Cities Transport Group, Department of Infrastructure Engineering, Melbourne School of Engineering, Centre for Disaster Management and Public Safety (CDMPS), The University of Melbourne, Parkville, VIC 3010, Australia

2 Smart Cities Transport Group, Department of Infrastructure Engineering, Melbourne School of Engineering, The University of Melbourne, Parkville, VIC 3010, Australia

3 Department of Infrastructure Engineering, Melbourne School of Engineering, Centre for Disaster Management and Public Safety (CDMPS), The University of Melbourne, Parkville, VIC 3010, Australia roads with maximum travel demand coverage are sorted as the most critical ones, for which a method-known as "link analysis"-is adopted. In an iterative process, first, a road with highest traffic volume is first labeled as "target link," and second, a portion of travel demand which is captured by the target link is excluded from travel demand. For the next iteration, the trimmed travel demand is then assigned to the network where all links including the target links run on the initial travel times. The process carries on until all links are labeled. The proposed methodology is applied to a largesized network of Winnipeg, Canada. The results shed light on also bottleneck points of the network which may warrant provision of additional capacity or parallel roads.

Keywords Critical roads · Achilles-heel roads · Sensor location problem $\cdot$ Flow-bundle $\cdot$ Link analysis

\section{Introduction}

Unexpected traffic disruptions and reliability consequences have made academia and the industry more interested in subjects such as resilience, reliability, vulnerability; flexibility, robustness, fragility and critical roads [1]. Although these concepts are yet to be unambiguously defined [2], each subject stands on its own merit representing some areas of concerns with a common denominator posed as the following key question: how does the transport system respond at such disruptive events? or "what is the damage, given that something happens" [3].

The disruption entails a wide spectrum of events from traffic accidents and incident to extreme events such as natural or man-made disasters. During and in the aftermath of such events, the most vital (or so-called critical) roads must be kept at a functioning level. 
Identifying a measure of robustness of the network at extreme events is a worthwhile effort which is also pursued in this study. In the first place, there is no consensus on the definition of network robustness much to the extent of the network stretched in a wide spectrum of disciplines [2, 4]. We adopt an intuitive definition provided by the Institute of Electrical and Electronics Engineers (1990): Robustness is the degree to which the network can function correctly in the presence of stressful conditions [1]. In other words, robustness is a characteristic of a network in withstanding or absorbing disturbances and remaining intact when exposed to disruption [4].

The roads are the backbone of a network based on which the operation is conducted. Irrespective of where we stand on the above-mentioned spectrum, the general approach to assessing the robustness (or inversely fragility) of the network is to find the critical roads, which is a daunting task. Critical roads could act like Achilles-heel that is that the entire network could become hostage to the disruption of few roads. In case of any incident leading to their closures, the network gets at a standstill. It is obvious that finding such Achilles-heel roads holds a central role to develop a mitigation scheme or to be prepared for any eventuality. Perhaps such findings may warrant investment in expanding the road capacity such as new roads, bridges or lane widening, a type of themes known as network design problem [5]. The criticalness degree of roads can also be looked at as an index to measure their importance which in turn is utilized in road constructions and their prioritization [6, 7]. At the other end of this spectrum, there might be a number of roads whose removal, in fact, improves network performance commonly known as Braess's paradox [8-10].

According to the findings in the literature, identifying such critical roads faces two main challenges: computational efficiency and theoretical development [11]. To cope with such difficulties, we proposed a heuristic method inspired by sensor (loop detector) location problem (SLP) [12]. The SLP basically stands for finding the minimum number and location of counting posts (in traffic count survey) in order to infer all traffic flows in a transport network. The widely accepted solution for the SLP is finding roads which can represent a broader range of origin-destination (OD) travel demand. This method is known as OD demand coverage based on which our heuristic method for identifying the Achilles-heel roads is developed. To this end, roads with maximum travel demand coverage are sorted as the most critical one, for which a method widely popular among practitioners known as "link analysis" is adopted. The proposed methodology is applied to a large-sized network of Winnipeg, Canada. The results shed light on also bottleneck points of the network which may warrant provision of additional capacity or parallel roads. These roads can also be considered as best possible locations for petrol station or police checkpoints since they represent the maximum number of vehicles passed through.

The impacts of the roads closure are simulated based on user-equilibrium traffic assignment for which the following assumptions-widely used in the literature-are made: (a) travel demand is fixed and quantified as a single matrix, (b) commuters have a perfect understanding of the travel time, (c) neither demand nor travel time changes over time. In other words, we solve for a static and deterministic traffic assignment problem (TAP) subject to a fixed travel demand. However, by relaxing one or some of the above-mentioned assignment-which resulted in stochastic, dynamic and variable travel demand methods-one can increase the realism and fidelity of the traffic simulation at the costs of additional computational times as well as some other computational complexities. Nevertheless, the consensus in the literature is to resort to the above-mentioned assumptions.

The rest of the article is organized as follows. Section 2 contains a literature review. Section 3 discusses the methodology. Section 4 presents numerical results of two case studies: Gao's test network and a large-size network of Winnipeg, Canada. Conclusion remarks are provided in Sect. 5 .

\section{Literature review}

In this section, we provide a review of the studies related to the concepts of vulnerability followed by the literature related to the SLP.

The subjects such as vulnerability, robustness, flexibility and resilience do not have a clear demarcation and definition [4]. Recent thorough reviews have been made by [4, 13-15]. Given the extensive breadth of the research, in the present review, we seek only the most recent takes of the literature on the subject of critical roads.

Most of the previous studies are conceptual methods on vulnerability lacking a holistic approach to quantifying and evaluating the vulnerability of transportation networks. Rosenkrantz et al. [16] suggest the idea of a "StructureBased Resilience Matrix" to measure the vulnerability/resilience of networks' components. Scott et al. [17] used network flows, link capacity and network topology to develop a network robust index measure. Leu et al. [18] used a network analysis to measure the robustness considering physical features of the network. Mattsson and Jenelius [19] provide an overview of recent research on vulnerability and the resilience of transport systems.

The consensus in the literature is to investigate the vulnerability based on the network topology [20, 21]. de Oliveira et al. [22] investigated two performance attributes of road networks, reliability and vulnerability and discuss 
the indicators found in the literature. The results show that the vulnerability indicators are more strongly affected by the characteristics of alternative routes. Aided by geography information systems (GIS), Kermanshah and Derrible [23] developed a data-driven approach to determining vulnerable locations in road networks with respect to land use information, demographic data and travel demand as well as some topological indicators. Similarly, Thekdi and Joshi [24] describe a scenario-based Bayesian approach to evaluate evidence from big-data resources, such as geographical landscape and demographic data, to identify vulnerable sections of the transportation network.

The plethora of the relevant studies can be divided into two groups: (a) A vast number of studies only consider topological characteristics of networks, such as accessibility, connectivity, shortest path [25-30]. What is missing in such approaches is the dynamic of the flow on the network. (b) Other studies are primarily concerned with the dynamic characteristic of flow such as commuters' route choosing behavior in the road network $[1,20,31]$. This study obviously belongs to the second group.

The critical roads sometimes are dealt with in the context of vulnerability or via resilience or through robustness and fragility. The consensus is to examine removals of the roads to find their impacts on the respective network. The roads are then attributed with an index based on which the critical ones are flagged [1, 32, 33]. Conforming to the aforementioned classification, there are also two major categories of indices to measure the overall performance of the network (also known as a measure of effectiveness): functional and topological [4]. The flow dynamic as an overall network performance index is largely formulated as changes in total travel time $[4,31]$.

Similarly, Jenelius et al. [3] elicited a number of link importance indices and site exposure indices based on the increase in generalized travel cost when links are closed. These measures are divided into two groups: one reflecting an "equal opportunities perspective" and the other a "social efficiency perspective" pertaining to the connectivity and weighted by travel demand, respectively. The generalized travel costs are measured based on the Dijkstra's shortest paths algorithm, and it is called "dynamic shortest path algorithm." In other words, the effects of the traffic congestions arising from the disruptions (road closures) are yet to be taken into account.

Albert et al. [34] investigated a class of in homogeneously wired networks called scale-free networks, which include the worldwide web, the Internet, social networks, cells as well as road networks. They found out that in response to random failures such networks exhibit an unexpected degree of robustness, to the extent their overall operations become unaffected. However, these networks are extremely vulnerable to targeted attacks aiming at a few links or nodes that play a vital role in maintaining the network's connectivity. A similar observation has also been reported by [35]. They have also displayed that malfunctioning of a single component of a network can generate a cascading effect, thus causing the entire network to collapse.

Wu et al. [36] have extensively studied the cascading effects of a number of failures' scenarios. In contrary to the previous studies, the congestion effects of the failures' scenarios are fully taken into account based on the userequilibrium (UE) traffic assignment. They displayed that two removal schemes flow-based and betweenness-based inflict the highest disruption compared to other removal scenarios (betweenness is an indicator of a node's centrality in a network, and it is equal to the number of shortest paths that pass through the respective node).

As the literature review indicates, though the road networks are resilient to random or natural failures, they are highly fragile to targeted attacks; that is why, we refer to it as Achilles-heel phenomenon.

The SLP has been found to be of the utmost difficult problems known as NP-hard [37-39]. The SLP has been of great interest to electrical engineering as well as computer science for which a number of different methods are presented [40-45]. Given the fact that SLP is NP-hard, the heuristic methods are deemed valid [46, 47]. Yang and Zhou [47] proposed four heuristic methods including maximal flow fraction, OD demand coverage. Some researchers consider geographical and/or topological disaggregation of link flows to place the sensors [38, 48, 49]. Zhang et al. [50] proposed a genetic algorithm hybridized with simulated annealing to find appropriate traffic count posts to monitor network's traffic flow. Viti et al. [41] provide a solution for the SLP by minimizing a measure of information loss of partial observability. Morrison and Martonosi [51] establish a necessary condition on the location of the sensors to enable the traffic flow to be computed. Larsson et al. [12] present a review of the solution methods of the SLP in the literature and appoint the OD demand coverage (ODDC) as a favorable method.

Based on the literature review, the following can be concluded. The road networks are shown to be of scale-free [52] that is there is a few, but a significant number of nodes with a lot of connections, whereas there are a high number of nodes with very few connections. This feature emerges from the fact that networks expand continuously by the addition of new nodes, and new nodes attach preferentially to nodes that are already well connected [53]. This is a clear definition of the road networks. Given the fact that the scale-free network is defenseless to targeted attacks, it is of the highest importance to flag these failures which are the mandate of the current study. To this end, the failure is defined as a number of drivers affected by targeted attacks 
which in turn calls on the SLP. There are a number of methods proposed for the SLP out of which the OD demand coverage is adopted in our research. Furthermore, we will discuss that the OD demand coverage implies the two most detrimental removal scenarios: flow-based and betweenness-based.

\section{Methodology}

Before proceeding to the next sections to discuss the phased methodology, let us introduce all the notations used in this article as presented in Table 1.

In this section based on the OD demand coverage criterion, a set of highly demanding roads are identified. To put it plainly, the candidate roads must be of highest importance. Such interpretation gives birth to a whole different problem in transportation in which traffic count posts for OD estimation (ODE) are sought. The task of ODE is centered on adjusting an outdated OD demand data based on a recently compiled traffic count of "some selected count posts." Obviously, the traffic count posts are placed on the road. Evidently, traffic survey and the count are a costly job, and hence, it is not possible to have traffic counts on all roads. Now the question to be first answered is: Which roads must be selected as count posts to be used in the ODE? It is also a valid answer to the SLP.

The ODDC can be mathematically formulated as follows [12]:

$[\mathrm{ODDC}] \max \sum_{r \in R} q_{r} \cdot \lambda_{r}$,

s.t. $\sum_{a \in A} \Pi_{a r} \cdot \mu_{a} \geq \lambda_{r} \quad r \in R$,

$\sum_{a \in A} \mu_{a}=\bar{n}$,

$\lambda_{r} \in\{0,1\}, \quad \mu_{a} \in\{0,1\}, \quad r \in R, \quad a \in A$,

where the road network is denoted by a set of links, $A$, and a set of OD pairs, $R$. The travel demand of OD pair $r \in R$ is represented by $q_{r}$. For every road in the network $a \in A$ and each OD pair $r \in R$, we consider binary decision variables $\mu_{a}$ and $\lambda_{r}$ : If a sensor is allocated, $\mu_{a}=1$ and $\mu_{a}=0$ otherwise; if sensors capture any trips pertaining to the respective OD pair, $\lambda_{r}=1$ and $\lambda_{r}=0$ otherwise. Furthermore, $\Pi_{a r}$ is a matrix of the size of $|A| \times|R|$ with binary entries: $\Pi_{a r}=1$ if traffic volume on $a \in A$ entails any trips pertaining to $r \in R$ and $\Pi_{a r}=0$ otherwise. The

Table 1 Notation glossary used in this manuscript

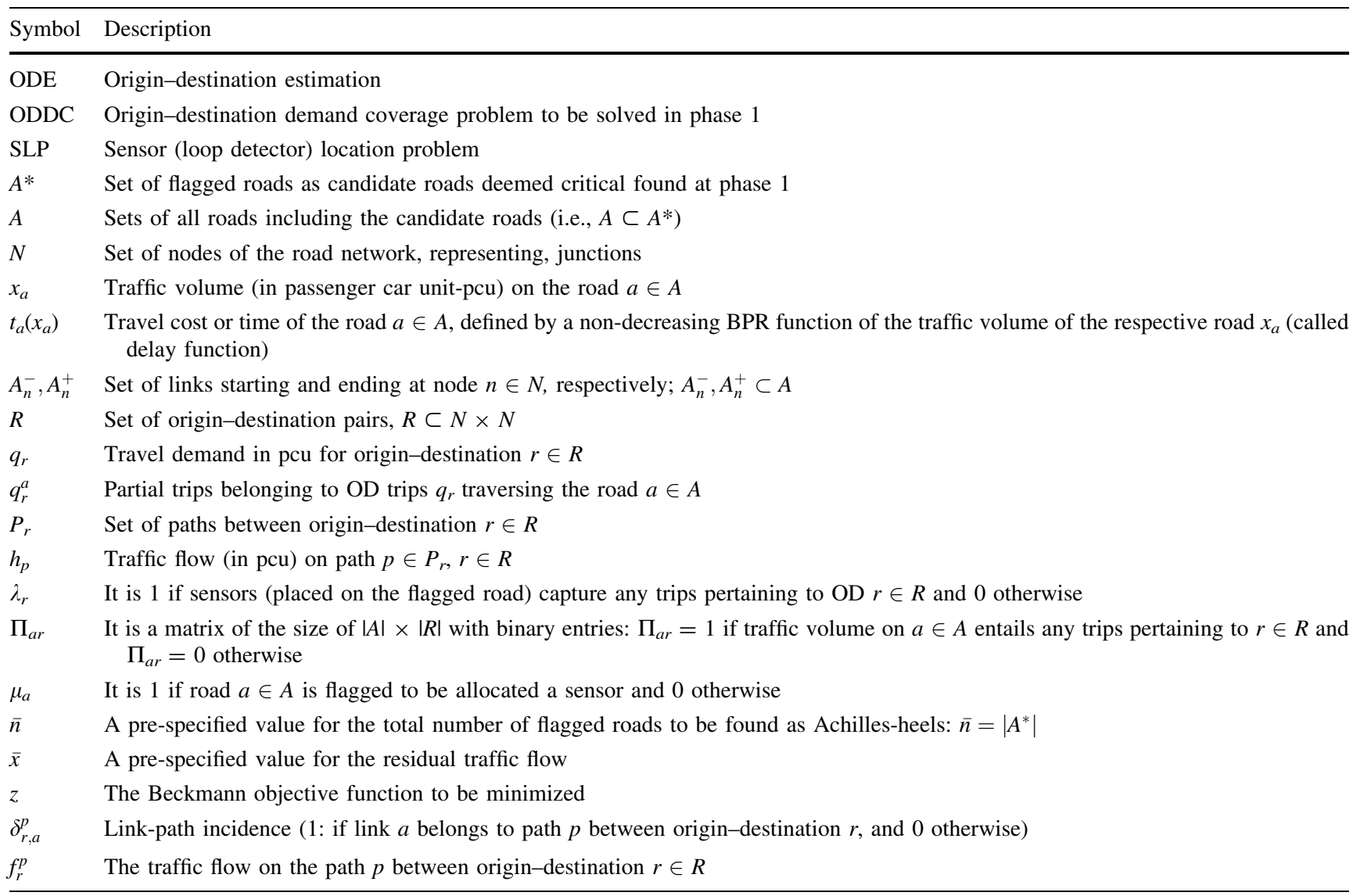


number of sensors to be allocated to roads is denoted by $\bar{n}$. In the end, a number of selected roads to be equipped with sensors constitute the set of flagged roads $A^{*} \subset A$ hence $\bar{n}=\left|A^{*}\right|$.

As discussed before, the SLP is proven to be NP-hard. In order to streamline such difficulty (especially in facing with real-sized networks), we adopt an intuitive and heuristic approach based on a popular practice among practitioners known as "select link analysis" or "flowbundle" [54] in which, traffic volume seen on a road is traced back and forth to its origins and destinations. In other words, a subset of the OD demand corresponding to the traffic volume of the respective road is first skimmed off and it is then highlighted in the traffic volume plot. By doing so, the trace of the target traffic volume will be shown on the network. The method is carried out in an iterative course. Given a network with the result of the traffic assignment, in each iteration, a road with highest traffic volume is flagged for the sensor. The flagged road is then subjected to the flow-bundle procedure to retrieve the corresponding OD trips, and we call it flagged trips. For the next iteration, the OD demand is first shaken off the flagged trips, and it is then used for a new traffic assignment (better to be called "partial traffic assignment").

The static, deterministic traffic assignment problem is traditionally formulated based on the Wardropian principles, that is, drivers choose the shortest paths:

$\min z(x)=\sum_{a \in A} \int_{0}^{x_{a}} t_{a}\left(x_{a}\right) \mathrm{d} x$,

s.t. : $\sum_{p} f_{r}^{p}=q_{r} \quad r \in R$,

$f_{r}^{p} \geq 0 \quad p \in P_{r}, \quad r \in R$,

$x_{a}=\sum_{i} \sum_{k} \sum_{p} f_{r}^{p} \cdot \delta_{r, a}^{p} \quad a \in A, p \in P_{r}, \quad r \in R$,

where $z$ the Beckmann objective function to be minimized; $f_{r}^{p}$ the traffic flow on the path $p$ between origin-destination $r \in R ; \delta_{r, a}^{p}$ a link-path incidence (1: if link $a$ belongs to the path $p$ between origin-destination $r$, and 0 otherwise) $[55,56]$. The rest of notations have already been introduced. The TAP is solved iteratively such that in each iteration links' travel times are updated based on the congestion level and it carries on until the difference of the values of the objective functions in two successive iterations becomes negligible.

Compared to the first traffic assignment effort, this new (partial) traffic assignment can be computed much more efficiently: Any solution algorithm for a TAP needs to start with a feasible solution (traffic volumes on roads for $\left.\left\{x_{a}, a \in A\right\}\right)$. In an iterative fashion, links' travel times $t_{a}$ and traffic volumes $x_{a}$ are updated until a satisfactory

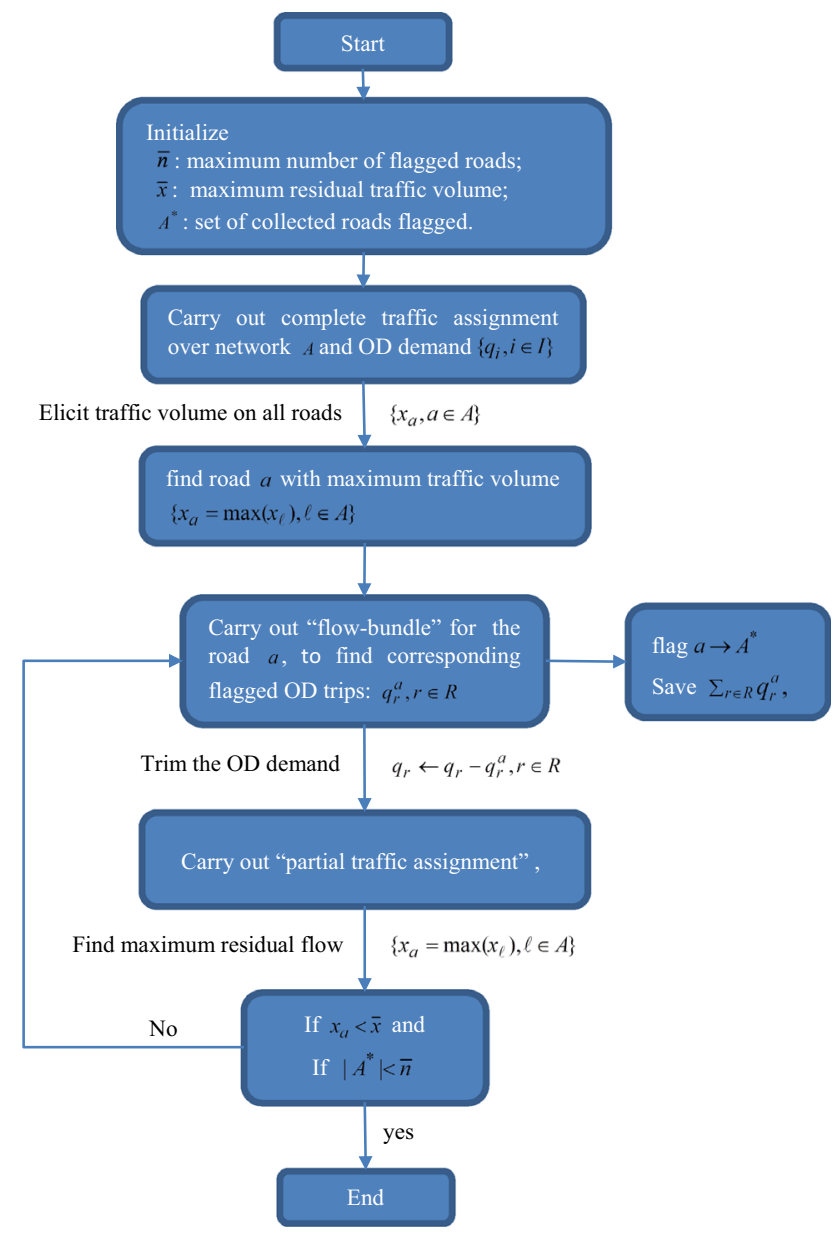

Fig. 1 Flow-bundle algorithm for the SLP

convergence criterion is met (the travel times are updated to also update the shortest paths).

For a new traffic assignment, in the absence of any information the algorithm initiates based on the free-flow travel time (called all-or-nothing traffic assignment). If there were a prior knowledge of the travel times close to those of the final optimal solution, then less number of iterations would be needed to meet the convergence criterion.

In the partial traffic assignment, this prior knowledge exists as discussed in the following. First, the $x_{a}$ from the previous traffic assignment is taken off from the OD travel demand, while the links' travel times remain intact which is also corresponding to those of the optimal solutions found in the first traffic assignment. This is because when a portion of trips is taken off the road network, we do not want to see any changes on drivers' routing behavior (still remaining in the road) for tracing the next round of trips. Hence, solving the new traffic assignment can be termed as "partial" traffic assignment. The process carries out until a certain number of flagged roads are obtained or residual traffic volume of the network becomes insignificant. An 
algorithmic exposition of the method is provided as follows (a pictorial presentation of the algorithm is also provided in Fig. 1):

Step 0: Set two target values for terminations: (a) $\bar{n}$ : number of flagged roads to allocate sensor, (b) $\bar{x}$ : maximum residual traffic volume. Initialize set $A^{*}$ designated to the roads flagged as selected for sensors. Given network $A$ and OD demand $\left\{q_{i}, i \in I\right\}$, carry out traffic assignment to get traffic volume on all roads denoted by $\left\{x_{a}, a \in A\right\}$;

Step 1: Find a road $a$ with maximum traffic volume (i.e., $\left.\left\{x_{a}=\max \left(x_{\ell}\right), \ell \in A\right\}\right)$ and add it to the flagged set $\left(a \rightarrow A^{*}\right)$. Carry out "flow-bundle" for the $\operatorname{road} a$, and find the corresponding flagged OD trips denoted by $q_{r}^{a}, r \in R$. Subtract the current OD demand from the flagged trips (i.e., $q_{r} \leftarrow q_{r}-q_{r}^{a}, r \in R$ ). Save $\sum_{r \in R} q_{r}^{a}$, as the ODCD of the road $a$. Now execute a partial traffic assignment.

Step 2: Find number of flagged roads and maximum residual traffic volume; if they are less than the target values $(\bar{x}$ and $\bar{n})$ and then go to step 1; otherwise, terminate the algorithm.

The outcomes of the algorithm are the flagged roads for the sensor. We specify values of the target number of flagged roads and maximum residual traffic volume $(\bar{x}$ and $\bar{n}$ ) in the undertaken case studies. By now the flagged roads are found. The order at which there are flagged as well as their corresponding OD coverage volumes shows their criticalness degree.

As the literature review indicates, the flow-based and betweenness-based removal schemes are more detrimental and disruptive to the networks' performance. The OD demand coverage method as implemented above (based on the link analysis) obviously bears traits of these two schemes. It is evidently flow-based because at each iteration the road with highest traffic flow is flagged and taken out of the network. Since a new traffic assignment problem is solved for each iteration and hence a new set of the shortest paths are calculated, the algorithm implicitly considers the betweenness (note that the betweenness refers to a total number of shortest paths passing through a respective node).

\section{Numerical evaluations}

In this section, we first examine the proposed methodology over a network similar to the Gao's 12-node network [57]. The Gao's network is readily available in the literature and is being used by the researchers as a common currency or a common benchmark network to exchange their findings. Therefore, the results reported in this paper can then be referred and examined in further studies. We then undertake a large-sized network of Winnipeg as a challenging case study to display applicability of the proposed methodology. All delay functions associated with the links conform to BPR-type.

As for the computational technology, we employ a desktop computer with Intel(R) Xeon(R) $3.70 \mathrm{GHz}$ and 64.0 GB RAM. The algorithm is coded in EMME 3 using "macro" the software's programming language.

Parameters setup of the algorithm is as follows:

- The relative gap needed by Frank-Wolfe algorithm to solve a traffic assignment is conservatively considered to be 0.0001 [58];

- As for the number of candidates in Phase 1 , one can carry out the process until the maximum traffic volume left on the roads becomes insignificant. For instance, the capacity of a local road can be a good criterion for being insignificant. Regardless, a pre-specified capped number can also be considered. For instance, for the Winnipeg case study, we intentionally extended the number of candidates to 100 flagged roads, by which the maximum traffic volume left on the network was found 50. By doing so, we wanted to challenge the performance of the proposed algorithm at such an extreme condition.

\subsection{Example 1: Gao's network}

Figure 2 illustrates the example network developed by Gao et al. [57], where the delay functions conform to $t_{a}=$ $\bar{t}_{a}+0.008 x_{a}^{4}$ (note: $\bar{t}_{a}$ is free-flow travel time and is shown in Fig. 2). The OD travel demand is also presented in Fig. 2 which is fairly different from the original Gao's network. The algorithm runs until the residual flow on the network becomes zero which results in 12 (out of 16) flagged roads.

The flagged roads are found as follows (start node-end node): 5-6, 7-8, 6-10, 1-5, 4-8, 3-7, 5-9, 8-12, 6-7, $10-11,2-6,2-3$; These roads are sorted in descending order based on their respective total demand coverage which are 6.28, 3.79, 3.34, 2, 2, 1.31, 1, 0.98, 0.91, 0.67, $0.24,0.07$. As can be seen, in early stages one can observe significant decreases in the demand coverages.

\subsection{Example 2: Winnipeg large-size network}

The large-sized road network of the city of Winnipeg, Canada, widely used in the literature [59] is undertaken for the numerical test here as well. This dataset has also been 


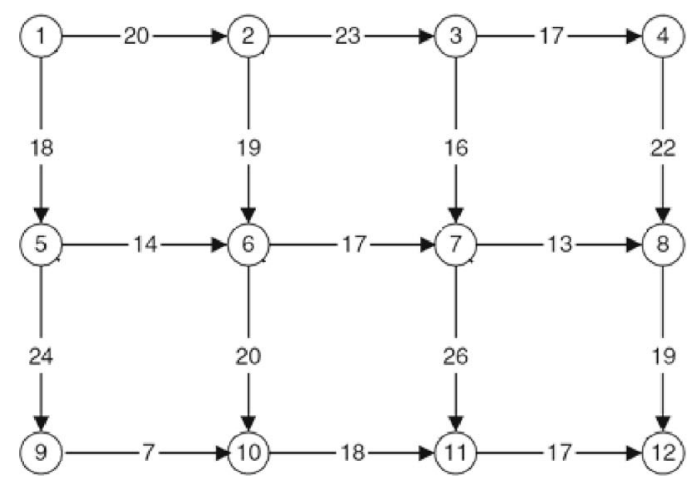

OD demand matrix:

\begin{tabular}{|c|c|c|c|c|c|}
\hline $\mathrm{O} \longrightarrow \mathrm{D}$ & 8 & 9 & 10 & 11 & 12 \\
\hline 1 & 1 & 1 & 1 & 1 & 1 \\
\hline 2 & 1 & 0 & 1 & 1 & 1 \\
\hline 3 & 1 & 0 & 0 & 1 & 1 \\
\hline 4 & 1 & 0 & 0 & 0 & 1 \\
\hline 5 & 1 & 1 & 1 & 1 & 1 \\
\hline
\end{tabular}

Remarks: values on the roads are free flow

travel time denoted by $\bar{t}_{a}$ in the delay function

Fig. 2 Gao's test network

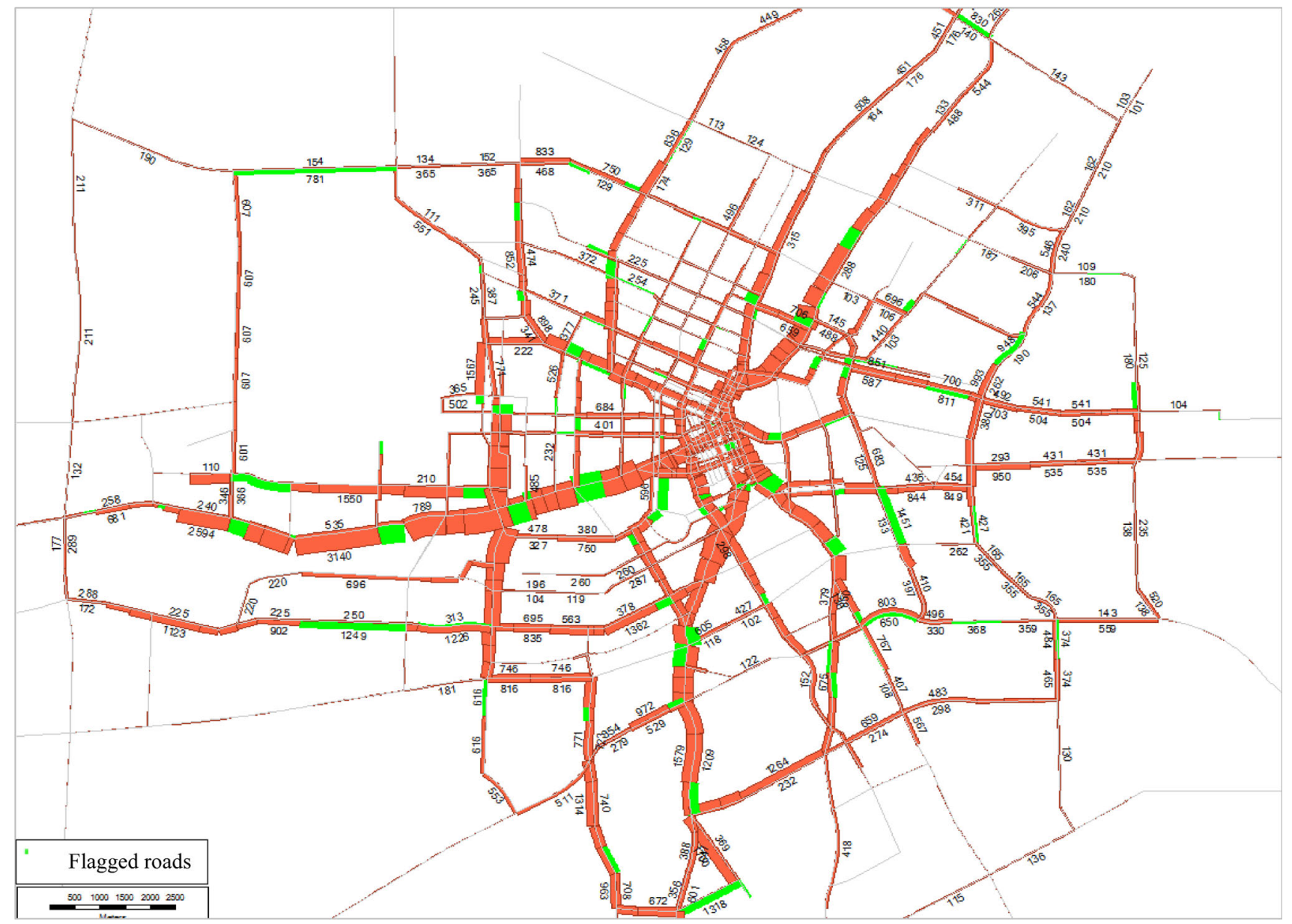

Fig. 3 Winnipeg network: locations of 100 roads found as flagged roads and traffic volume of the no-disruption scenario

provided in the EMME 3 [60]. The road network is comprised of 154 zones, 943 nodes and 3075 directional links. Given a high number of roads, the algorithm runs for 100 iterations to find 100 flagged roads.

Figure 3 along with Table 2 shows the locations and characteristics of the flagged roads. In addition to the OD coverage, other characteristics of the roads including freeflow speed (ffs), length, capacity, free-flow travel time ( $t 0)$ and travel time are also provided in Table 2. As can be seen in Fig. 3, the critical roads are largely flagged on the main roads and roads surrounding the CBD.

Figure 4 depicts how OD demand coverages vary over a descending order of the flagged critical roads. The figure shows a deep reduction in the first 10 roads and a very slight slope in the rest of the roads. Hence, these 10 roads must be pinpointed for further investigations and actions 


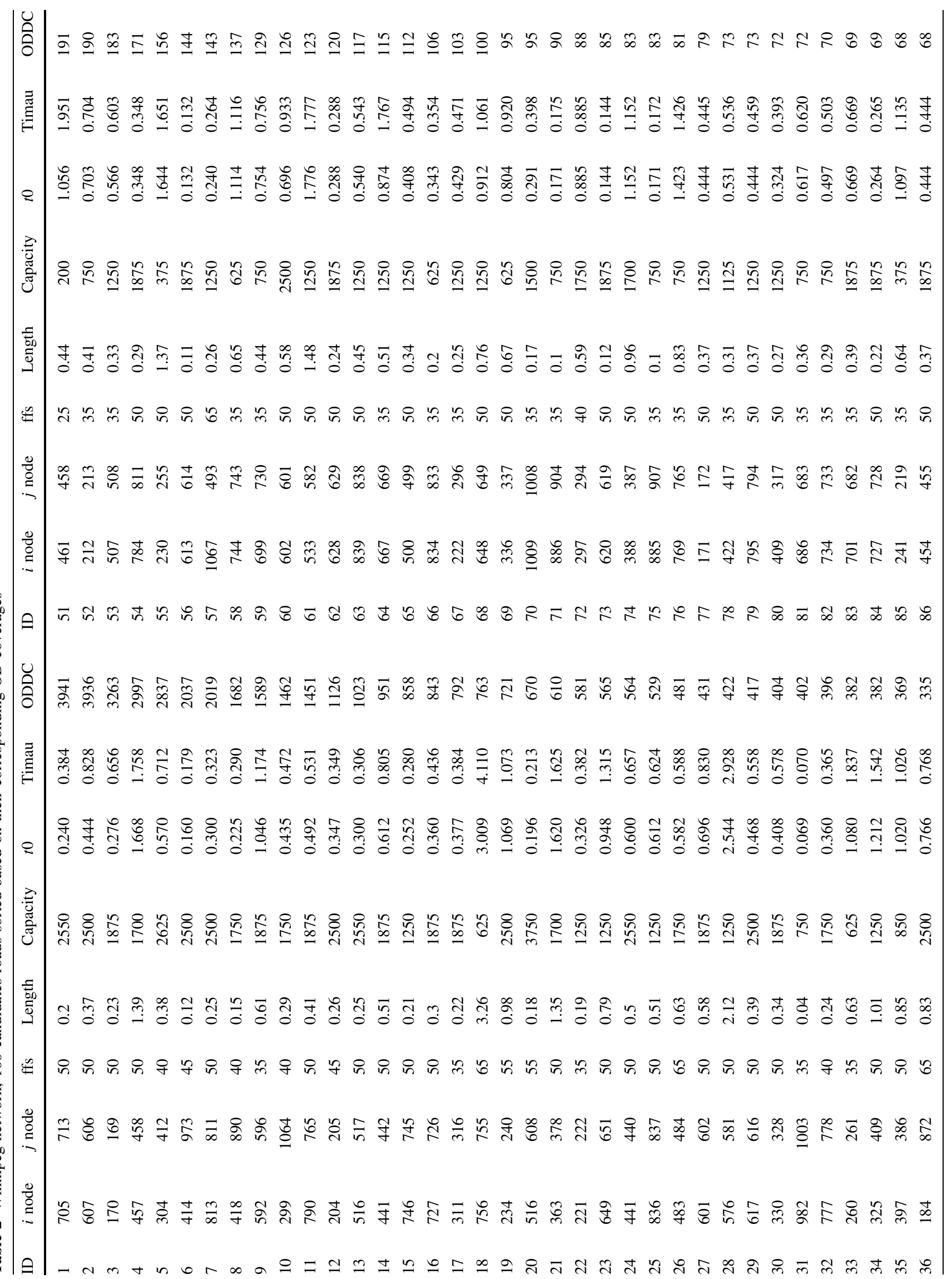




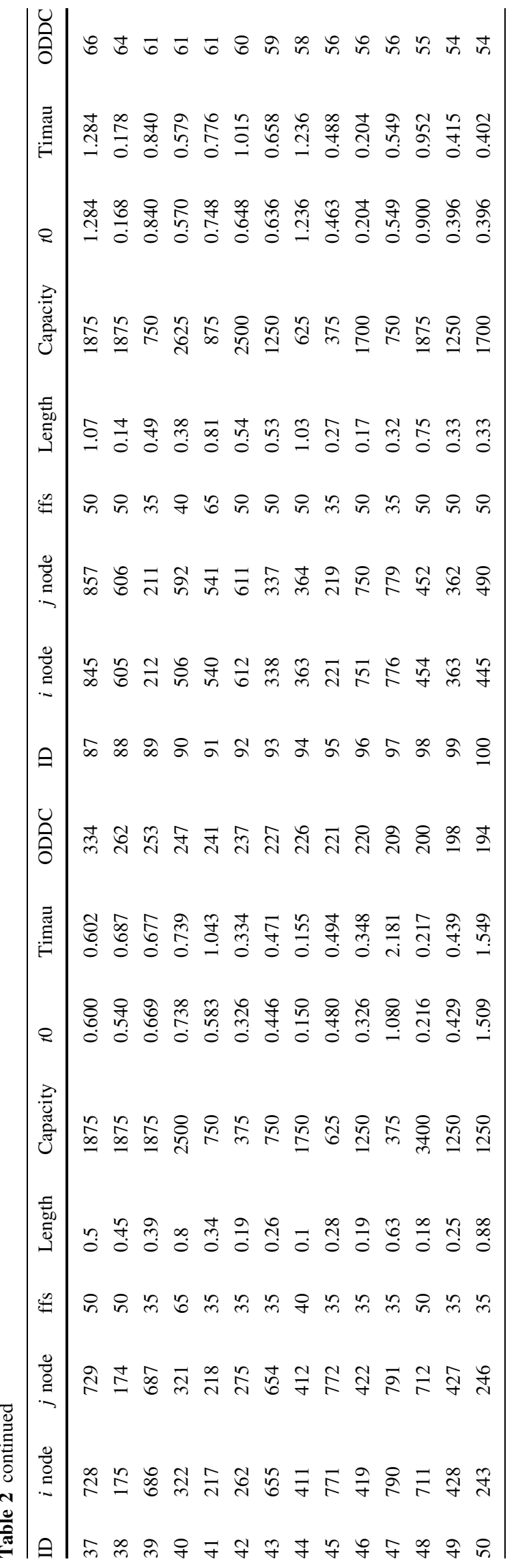

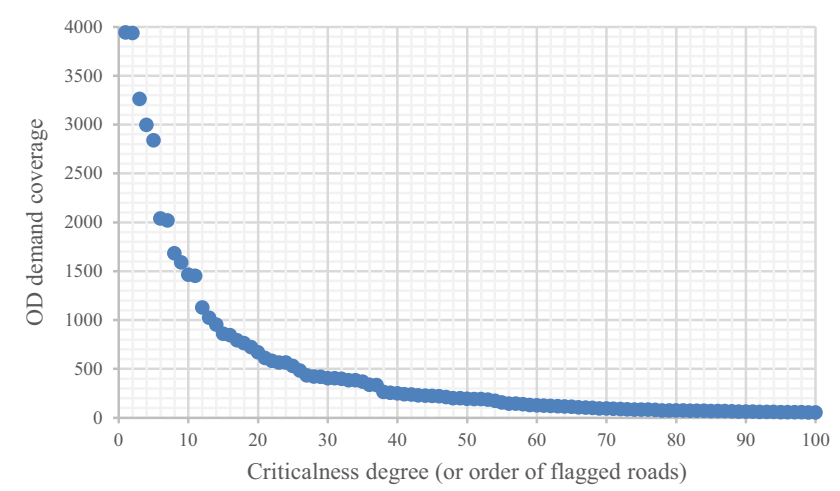

Fig. 4 Winnipeg test, a variation of OD demand coverage over the flagged roads

such as preparing mitigation plans, reinforcement measures and.

\section{Conclusions}

In this study, we developed a heuristic methodology to find the most critical roads whose closures are devastating as if they act like Achilles-heel, and bring the entire network to a halt. The Achilles-heels is unfortunately in the genes of the road networks since they are proven to be of the scalefree networks that are the networks highly vulnerable to selected and targeted disruptions or attacks.

To this end, a set of roads which represent the travel demand the most are deemed to be critical are flagged. To do so, we borrowed the notion of sensor (loop detector) location problem which is proven to be of utmost difficult problems in terms of computational expenses. Such difficulties convinced us to resort to a heuristic methodology based on the concept of the maximum OD coverage which is already found as a favorable method for the SLP in the literature.

According to the OD coverage method, highly demanding roads (those that cover the travel demand the most) are found, for which we employed "link analysis": method-a popular tool for practitioner in traffic impact studies. We applied the algorithm to the Gao's test network and a real network of Winnipeg, Canada.

The main contribution of this study can be attributed to the way the OD coverage method is implemented in which the two most detrimental removal scenarios (i.e., flowbased and betweenness-based) are considered in one go. Moreover, in terms of the computational expenses, the proposed methodology provides highly efficient methods much owed to the adoption of the partial traffic assignment.

The results can be found of the highest importance to the traffic authorities in their quest to protect the road networks against targeted disruptions which would have cascading disruption across the system. 
The subjects such as critical roads, robustness and resilience are seeing interest from different stakeholders such as authorities, practitioners and academia. Knoop et al. [33] have reviewed a variety of research and have assessed the quality of the outcomes. They found there is no consistency in the outcome. Such inconsistency and uncertainty have also reported by others [13]. Future studies can direct to first establish a consensus in definitions of these themes and their implications. The authors are currently studying on a global index to measure the robustness (or adversely fragility) of the network. Similar indices can be developed for other subjects. We are also working to find critical scenarios (rather than critical roads) which might consist of critical and non-critical roads. In other words, there can be some non-critical roads; if they become closed at the same time, the network becomes a gridlock Bagloee et al. [61].

Acknowledgements The authors are indebted to Prof. Zhao and two anonymous reviewers whose remarks contributed to improving the quality and the clarity of the final version.

Open Access This article is distributed under the terms of the Creative Commons Attribution 4.0 International License (http:// creativecommons.org/licenses/by/4.0/), which permits unrestricted use, distribution, and reproduction in any medium, provided you give appropriate credit to the original author(s) and the source, provide a link to the Creative Commons license, and indicate if changes were made.

\section{References}

1. Nagurney A, Qiang Q (2012) Fragile networks: identifying vulnerabilities and synergies in an uncertain age. Int Trans Oper Res 19(1-2):123-160

2. Maltinti F, Melis D, Annunziata F (2011) Road network vulnerability: a review of the literature. In: Chong WKO, Hermreck $\mathrm{C}$ (eds) The international conference on sustainable design and construction (ICSDC). Integrating sustainability practices in the construction industry, March 23-25, Kansas City, Missouri

3. Jenelius E, Petersen T, Mattsson L-G (2006) Importance and exposure in road network vulnerability analysis. Transp Res Part A Policy Pract 40(7):537-560

4. Faturechi R, Miller-Hooks E (2014) Travel time resilience of roadway networks under disaster. Transp Res Part B Methodol 70:47-64

5. Bagloee SA, Tavana M, Ceder A, Bozic C, Asadi M (2013) A hybrid meta-heuristic algorithm for solving real-life transportation network design problems. Int $\mathrm{J}$ Logist Syst Manag 16(1):41-66

6. Bagloee SA, Tavana M (2012) An efficient hybrid heuristic method for prioritising large transportation projects with interdependent activities. Int J Logist Syst Manag 11(1):114-142

7. Bagloee SA, Asadi M (2015) Prioritizing road extension projects with interdependent benefits under time constraint. Transp Res Part A Policy Pract 75:196-216

8. Bagloee SA, Ceder A, Tavana M, Bozic C (2013) A heuristic methodology to tackle the Braess Paradox detecting problem tailored for real road networks. Transp A Transp Sci 10(5):437-456
9. Braess D, Nagurney A, Wakolbinger T (2005) On a paradox of traffic planning. Transp Sci 39(4):446-450

10. Braess D (1968) Über ein Paradoxon aus der Verkehrsplanung. Unternehmensforschung 12(1):258-268

11. Taylor MA (2012) Network vulnerability in large-scale transport networks. Transp Res Part A Policy Pract 46(5):743-745

12. Larsson T, Lundgren JT, Peterson A (2010) Allocation of link flow detectors for origin-destination matrix estimation-a comparative study. Comput-Aided Civil Infrastruct Eng 25(2):116131

13. Reggiani A, Nijkamp P, Lanzi D (2015) Transport resilience and vulnerability: the role of connectivity. Transp Res Part A Policy Pract 81:4-15

14. Wang Z, Chan AP, Yuan J, Xia B, Skitmore M, Li Q (2014) Recent advances in modeling the vulnerability of transportation networks. J Infrastruct Syst 21(2):2-9

15. Bagloee SA, Asadi M, Richardson L (2011) Identifying traffic count posts for origin-destination matrix adjustments: an approach to actual size networks. J Transp Manag 22(1):79-88

16. Rosenkrantz DJ, Goel S, Ravi S, Gangolly J (2004) Structurebased resilience metrics for service-oriented networks, pp 345-362

17. Scott DM, Novak DC, Aultman-Hall L, Guo F (2006) Network robustness index: a new method for identifying critical links and evaluating the performance of transportation networks. J Transp Geogr 14(3):215-227

18. Leu G, Abbass H, Curtis N (2010) Resilience of ground transportation networks: a case study on Melbourne. In: 33rd Australasian transport research forum conference canberra, Australia

19. Mattsson L-G, Jenelius E (2015) Vulnerability and resilience of transport systems-a discussion of recent research. Transp Res Part A Policy Pract 81:16-34

20. Jenelius E, Mattsson L-G (2015) Road network vulnerability analysis: conceptualization, implementation and application. Comput Environ Urban Syst 49:136-147

21. Kermanshah A, Karduni A, Peiravian F, Derrible S (2014) Impact analysis of extreme events on flows in spatial networks, pp 29-34

22. de Oliveira EL, da Silva Portugal L, Junior WP (2016) Indicators of reliability and vulnerability: similarities and differences in ranking links of a complex road system. Transp Res Part A Policy Pract 88:195-208

23. Kermanshah A, Derrible S (2016) A geographical and multicriteria vulnerability assessment of transportation networks against extreme earthquakes. Reliab Eng Syst Saf 153:39-49

24. Thekdi SA, Joshi NN (2016) Risk-based vulnerability assessment for transportation infrastructure performance. Int J Crit Infrastruct 12(3):229-247

25. Callaway DS, Newman ME, Strogatz SH, Watts DJ (2000) Network robustness and fragility: percolation on random graphs. Phys Rev Lett 85(25):5468-5471

26. Boccaletti S, Latora V, Moreno Y, Chavez M, Hwang D-U (2006) Complex networks: structure and dynamics. Phys Rep 424(4):175-308

27. Newman M, Barabasi A-L, Watts DJ (2006) The structure and dynamics of networks. Princeton University Press, Princeton

28. Newman ME (2003) The structure and function of complex networks. SIAM Rev 45(2):167-256

29. Tu Y, Yang C, Chen X (2012) Road network topology vulnerability analysis and application. Proc ICE-Transp 166(2):95-104

30. Zhang H, Fata E, Sundaram S (2015) A notion of robustness in complex networks. IEEE Trans Control Netw Syst 2(3):310-320

31. Luathep P (2011) Stochastic transport network model and optimization for reliability and vulnerability analysis. Ph.D. dissertation, The Hong Kong Polytechnic University

32. El-Rashidy RA, Grant-Muller SM (2014) An assessment method for highway network vulnerability. J Transp Geogr 34:34-43 
33. Knoop VL, Snelder M, van Zuylen HJ, Hoogendoorn SP (2012) Link-level vulnerability indicators for real-world networks. Transp Res Part A Policy Pract 46(5):843-854

34. Albert R, Jeong H, Barabási A-L (2000) Error and attack tolerance of complex networks. Nature 406(6794):378-382

35. Crucitti P, Latora V, Marchiori M, Rapisarda A (2004) Error and attack tolerance of complex networks. Phys A 340(1):388-394

36. Wu J, Gao Z, Sun H (2007) Effects of the cascading failures on scale-free traffic networks. Phys A 378(2):505-511

37. Bianco L, Confessore G, Gentili M (2006) Combinatorial aspects of the sensor location problem. Ann Oper Res 144(1):201-234

38. Bianco L, Confessore G, Reverberi P (2001) A network based model for traffic sensor location with implications on O/D matrix estimates. Transp Sci 35(1):50-60

39. Chin F, Chrobak M, Yan L (2014) Algorithms for placing monitors in a flow network. Algorithmica 68(1):1-15

40. Hu S-R, Liou H-T (2014) A generalized sensor location model for the estimation of network origin-destination matrices. Transp Res Part C Emerg Technol 40:93-110

41. Viti F, Rinaldi M, Corman F, Tampère CM (2014) Assessing partial observability in network sensor location problems. Transp Res Part B Methodol 70:65-89

42. Bianco L, Cerrone C, Cerulli R, Gentili M (2014) Locating sensors to observe network arc flows: exact and heuristic approaches. Comput Oper Res 46:12-22

43. Castillo E, Grande Z, Calviño A, Szeto WY, Lo HK (2015) A state-of-the-art review of the sensor location, flow observability, estimation, and prediction problems in traffic networks. J Sens. doi: $10.1155 / 2015 / 903563$

44. Gentili M, Mirchandani P (2012) Locating sensors on traffic networks: models, challenges and research opportunities. Transp Res Part C Emerg Technol 24:227-255

45. Fu C, Zhu N, Ling S, Ma S, Huang Y (2016) Heterogeneous sensor location model for path reconstruction. Transp Res Part B Methodol 91:77-97

46. Lam W, Lo H (1990) Accuracy of OD estimates from traffic counts. Traffic Eng Control 31(6):358-367

47. Yang H, Zhou J (1998) Optimal traffic counting locations for origin-destination matrix estimation. Transp Res Part B Methodol 32(2):109-126
48. Ehlert A, Bell MG, Grosso S (2006) The optimisation of traffic count locations in road networks. Transp Res Part B Methodol 40(6):460-479

49. Yang H, Yang C, Gan L (2006) Models and algorithms for the screen line-based traffic-counting location problems. Comput Oper Res 33(3):836-858

50. Zhang J, Zhang X, Wu J (2007) Genetic simulated annealing algorithm for optimal deployment of flow monitors, pp 398-402

51. Morrison DR, Martonosi SE (2015) Characteristics of optimal solutions to the sensor location problem. Ann Oper Res 226(1):463-478

52. Lämmer S, Gehlsen B, Helbing D (2006) Scaling laws in the spatial structure of urban road networks. Phys A 363(1):89-95

53. Barabási A-L, Albert R (1999) Emergence of scaling in random networks. Science 286(5439):509-512

54. Bar-Gera H, Boyce D, Nie Y (2012) User-equilibrium route flows and the condition of proportionality. Transp Res Part B Methodol 46(3):440-462

55. Sheffi Y (1985) Urban transportation networks: equilibrium analysis with mathematical programming methods. Prentice-Hall, Englewood Cliffs

56. Patriksson P (1994) The traffic assignment problem: models and methods, VSP BV, The Netherlands. Facsimile reproduction published in 2014 by Dover Publications, Inc., Mineola, New York, NY

57. Gao Z, Wu J, Sun H (2005) Solution algorithm for the bi-level discrete network design problem. Transp Res Part B Methodol 39(6):479-495

58. Boyce D, Ralevic-Dekic B, Bar-Gera H (2004) Convergence of traffic assignments: how much is enough? J Transp Eng 130(1):49-55

59. Bar-Gera H (2016) Transportation network test problems, December 1, 2016. http://www.bgu.ac.il/ bargera/tntp/

60. INRO (2009) EMME3 v 3.2. EMME3 User's Guide

61. Bagloee SA, Sarvi M, Wolshon B, Dixit V (2017) Identifying critical disruption scenarios and a global robustness index tailored to real life road networks. Transp Res Part E: Logist Transp Rev 98:60-81 